\title{
Leibniz: medicina, salud y epidemias
} Leibniz: Medicine, Health and Epidemics

\author{
Álvaro Carvajal Villaplana \\ Universidad de Costa Rica \\ alvaro.carvajal@ucr.ac.cr
}

Resumen: En 2020, una epidemia ha puesto en peligro la existencia de la humanidad; el protagonista es el coronavirus SARS-CoV-2 que produce la enfermedad de la COVID-19. En la historia de la humanidad no es la primera vez que esto sucede, pero dado la complejidad de las sociedades actuales, y en razones de los medios de comunicación, así como la integración global, sus efectos se dimensionan. Leibniz estudió estas epidemias, y de sus observaciones de los casos, extrajo una serie de recomendaciones, y elaboró una especie de teoría de salud pública sobre cómo enfrentar una pandemia. En este artículo se ofrece una explicación de dicha propuesta, enmarcada en el contexto más amplio de su sistema filosófico a partir del principio de vida, su concepción de la salud como un bien común y el principio de justicia que debe regir la protección de los(as) ciudadanos(as).

Palabras clave: Leibniz, salud, epidemias, COVID-19, bien común. 
Abstract: In 2020 a pandemic is threatening the lives of human beings everywhere; the agent is the coronavirus SARS-CoV-2 and the result is the disease known as COVID-19. This is not the first time in history that something like this happens, but as a result of the complexity of human interactions in presentday society and the interconnectedness of communications in globalization, the consequences acquire a new dimension. Epidemics were not unknown to Leibniz, who proposed recommendations from his observations of cases. He devised a theory of public health, helpful at facing an epidemic. In this paper we present an explanation of such a proposal, within the wider frame of his philosophical system, where we find a life principle, a conception of health as common good and a principle of justice from which the protection of all citizen is derived.

Keywords: Leibniz, health, epidemics, COVID-19, common good.

Fecha de recepción: $27 / 7 / 2020$

Fecha de aceptación: 17/2/2021

Carvajal Villaplana, Álvaro (2021). «Leibniz; medicina, salud y epidemias». Monograma. Revista Iberoamericana de Cultura y Pensamiento, $\mathrm{n}^{\circ}$ 8, pp. 131-160. doi: 10.36008/monograma.2021.08.2003. http: revistamonograma.com. ISSN: 2603-5839. 


\section{Introducción}

Leibniz es un filósofo que no deja de sorprender, su pensamiento se adelantó a muchas situaciones contemporáneas. En el actual momento crucial para la humanidad, a partir de la crisis sanitaria global provocada por la COVID-19, podemos encontrar en Leibniz un pensamiento que ayuda a comprender la crisis, dos aspectos resaltan: a) la importancia de la salud para la vida humana, para la felicidad y el progreso; y b) la propuesta de una teoría para la contención de una pandemia o peste. Él auguró una serie de medidas y acciones para evitar el contagio, la enfermedad y la muerte por una peste, sus propuestas no se diferencian sustancialmente de lo propuesto por las actuales teorías de la salud pública. Así, Leibniz en el siglo XVII, ya sabía qué se debía hacer.

Las ideas de Leibniz sobre la salud, la vida y la muerte se encuentran en una diversidad de escritos de su extensa obra. Para este artículo se realizó un recorrido por su obra, con el afán de ubicar, organizar e interpretar sus ideas sobre la salud, no así sobre la vida y la muerte, ya que estos temas los abordé en otros artículos ${ }^{1}$. En cambio, su pensamiento sobre las pandemias y las medidas de control, se encuentran sistematizadas en la Carta de Leibniz al Duque Ernst-Augusto, de 1679, la cual aparece en la colección de textos de Jaime de Salas, Escritos de filosofía jurídica y política (2011).

1 En Carvajal Villaplana, Álvaro; (2019) «Leibniz: argumentos para la defensa de los animales». En Carvajal, Álvaro (ed.) Leibni\%: Máquinas inteligentes, multiculturalismo y ética de la vida. Granada, España: Comares: 45-69. Y en 2016, «Muerte, sufrimiento y mal en Leibniz». En NicoLÁs, J. A. et al., La monadología de Leibniz a debate. Granada, España: Comares: 225-238.

Carvajal Villaplana, Álvaro (2021). «Leibniz; medicina, salud y epidemias». Monograma. Revista Iberoamericana de Cultura y Pensamiento, n. ${ }^{\circ} 8$, pp. 131-160. doi: 10.36008/monograma.2021.08.2003. http: revistamonograma.com. ISSN: 2603-5839. 
Puede decirse que Leibniz presenta una teoría de la salud pública para la contención de una epidemia, tal teoría tiene su base en la ética y la política, ya que él considera que es indispensable que el gobernante asuma la responsabilidad de la prevención de una pandemia para no lamentar las consecuencias de no actuar oportunamente.

Para Leibniz la vida es sumamente importante, por eso elaboró sus principios de vida (Véase, Carvajal, 2019; Nicolás, 2013)²; a partir de este principio, aquí se considera que la salud adquiere relevancia para Leibniz. La salud es tan valiosa como la razón, es a lo que las ciencias deben orientarse. Además, porque la salud es un bien común, y conforma parte de la aspiración de obtener la felicidad. El tema de la salud se relaciona con la idea de los mundos posibles. La salud se contrasta con la enfermedad y el mal.

\section{Saludy enfermedad: entre la perfección y el sufrimiento}

Leibniz asume que la salud es un asunto que no puede menospreciarse, ni descuidarse por asuntos insignificantes, ni puede dejarse a la desidia. Para él, la salud se encuentra al mismo nivel de importancia que la razón, la república, la guerra y la paz, tal como lo expresa en la siguiente cita:

...pero cabe reprochar al género humano que deje habitualmente al acaso algunos asuntos importantísimos mientras atiende, en cambio, con eficacia a asuntos insignificantes. Así pues, debe seguirse el ejemplo de los jurisconsultos que colocan a la razón junto a las más serias deliberaciones [213] sobre la vida y la salud, la república, los asuntos de la guerra y de la paz [...] $(1669 / 2011: 431)^{3}$

2 Véase Consideraciones sobre los principios de vida y sobre las naturalezas plásticas, por el autor del sistema de la armonía preestablecida (1705, en Echeverría, 2009).

3 Para una balanza del derecho que permita apreciar los grados de las pruebas y de las probabilidades, por

Carvajal Villaplana, Álvaro (2021). «Leibniz; medicina, salud y epidemias». Monograma. Revista Iberoamericana de Cultura y Pensamiento, $\mathrm{n}^{\circ}$ 8, pp. 131-160. doi: 10.36008/monograma.2021.08.2003. http: revistamonograma.com. ISSN: 2603-5839. 
Igualmente, en la misma línea del argumento anterior, de la siguiente cita se desprende que la negligencia de los hombres, no ha de afectar la conservación de la salud, la cual ubica al mismo nivel de nivel que otros bienes:

...y creo que si lo principal, de lo que depende todo lo demás, estuviera recogido con orden, se asombrarían los hombres de sus riquezas tanto como de su propia negligencia. Sería posible entonces superar gran cantidad de males y procurar infinidad de comodidades de la vida, y sobre todo se encontraría muy a menudo el medio de conservar la salud y de aumentar nuestras perfecciones, que debe ser el fin principal de todos nuestros estudios [...] (1686/1999: 5) ${ }^{4}$

Por otra parte, la salud se contrasta con la enfermedad, esta última consiste en una situación de vulnerabilidad, la que perjudica «nuestra fuerza de actuar», de tal manera que la salud es indispensable para la acción humana, es una fuerza que se relaciona con la libertad para actuar; tal como lo expresa en la siguiente cita: «Y así como la enfermedad proviene de una acción vulnerada, como bien han observado los entendidos en medicina, así por otra parte la perfección se muestra en la fuerza para actuar, ya que todo ser consiste en una cierta fuerza, y a mayor fuerza, más elevado y libre es el ser» (1694-1698/2003c: 457).

Además, opone la perfección del ser con la degradación que produce la enfermedad, ya que la salud produce el equilibrio para llegar a la perfección, la salud, aquí no es un fin, sino un medio para la perfección, la cual consiste en la felicidad. Apréciese la siguiente cita: «Llamo perfección a todo enaltecimiento del ser;

Gottfried Veran de Lublin; este último es un seudónimo a los que recurrió Leibniz (Olaso, 2011, nota 1: 428).

4 La organización del conocimiento y la felicidad del género humano (en Andreu, 1999; según la nota de Andreu, los manuscritos sobre la ciencia universal datan de 1686).

Carvajal Villaplana, Álvaro (2021). «Leibniz; medicina, salud y epidemias». Monograma. Revista Iberoamericana de Cultura y Pensamiento, n. ${ }^{\circ}$, pp. 131-160. doi: 10.36008/monograma.2021.08.2003. http: revistamonograma.com. ISSN: 2603-5839. 
pues, así como la enfermedad es un cierto modo una degradación y una caída desde la salud, así la perfección es algo que se alza por sobre la salud; la salud misma empero se halla en el medio y en equilibrio y sienta las bases de la perfección» (1694-1698/2003c: $457)^{5}$. En el mismo sentido, en el escrito De la felicidad afirma que la sabiduría no es otra cosa que la ciencia de la felicidad, que nos enseña a alcanzar la felicidad (1694-1698/2003c: 455).

La salud no es solo una enfermedad que afecta al cuerpo en el aspecto físico, en su texto De la sabiduría (1694-1698), vincula la salud con el estado de ánimo, con los afectos, que pueden conducir a problemas de salud, por ejemplo, se refiere a la alegría intempestiva y desmesurada, como «[...] cuando nuestro placer y nuestra fuerza se manifiestan en aquellas acciones en virtud de las cuales se debilitan otras fuerza y acciones elevadas» (1694-1698/2003a: 461), y cita la metáfora del caso de Eva, que disfruta con suma alegría de una manzana que luego resulta ser un veneno y produce amargura, lo cual conduce a daños en la salud y el entendimiento. De esta manera reitera que la salud consiste en equilibrio, y que los excesos pueden conducir a la desmejora de la salud.

Tal parece que para Leibniz la salud y la enfermedad son hechos relacionales, puesto que, para apreciar la salud, es necesario conocer o vivir la enfermedad, la última permite contar con una mayor valoración del bien que produce la salud. En un texto central del sistema leibniziano, como es la Teodicea, se pregunta: «[...] ¿nos agrada bastante la salud, y damos por ello suficientes gracias a Dios aún no habiendo estado nunca enfermos? ¿y no es necesario con mucha frecuencia que un poco de mal haga al bien más sensible, es decir, lo haga mayor?»

5 De la felicidad (1694-1698, en Andreu, 1999).

Carvajal Villaplana, Álvaro (2021). «Leibniz; medicina, salud y epidemias». Monograma. Revista Iberoamericana de Cultura y Pensamiento, n. ${ }^{\circ}$ 8, pp. 131-160. doi: 10.36008/monograma.2021.08.2003. http: revistamonograma.com. ISSN: 2603-5839. 
(1710/2012: 102-103). De lo antecedente, se desprende que la enfermedad, en Leibniz, está vinculada con la noción de mal y como veremos más adelanta con la idea de mundos posibles.

En la Teodicea, Leibniz se refiere al pecado, si bien la enfermedad puede verse como un pecado, más claramente se le puede identificar como un $\mathrm{mal}^{6}$; por lo que interesa destacar la idea de que de un mal puede surgirse un bien. En De la verdadera teología mística (1697-1698), se reafirma tal idea: «Dios ha permitido el pecado, porque sabía que de un mal se puede sacar un bien mayor, solo los males pierden por el pecado, pero la creación en su conjunto no ha perdido con ello, sino ganado» (1694-1698/2003b: 452), ya que - parafraseando a Leibniz - la predestinación procede originalmente de la previsión de los méritos de los hombres, no por la fe provista ni las obras previstas, pues lo que el ser humano haga de bien, le viene predicho por Dios. En todo caso, la enfermedad puede verse como parte del pecado en cuanto que los hombres no hacen todo lo debido para procurar la salud, ya sea por negligencia o por descuido de los asuntos importantes.

Igualmente, en la Teodicea plantea que de dos males puede salir un bien, aplicado a la enfermedad puede entenderse que de ella pueden obtenerse cosas buenas. En este sentido, cabe destacar su idea de que la salud es un bien que se reconoce como tal, si se conoce a la enfermedad. Pero no es que la enfermedad sea algo deseado, puesto que Dios quiere la salud para todas las personas, en sus palabras:

...pues una cosa pequeña añadida a otra cosa pequeña puede a menudo producir algo mejor que la combinación de otras dos,

$6 \mathrm{El}$ tema del mal no es posible trabajarlo en este artículo, pero puede consultarse a Cardona, en Dolor en la armonía. Justificación leibniziana del sufrimiento (2012) y Robinet, en Justicia y terror. Leibniz. y el principio de la razón (2005); también Roldán (3013) y Carvajal (2016).

Carvajal Villaplana, Álvaro (2021). «Leibniz; medicina, salud y epidemias». Monograma. Revista Iberoamericana de Cultura y Pensamiento, n. ${ }^{\circ} 8$, pp. 131-160. doi: 10.36008/monograma.2021.08.2003. http: revistamonograma.com. ISSN: 2603-5839. 
cada una de las cuales por sí misma es preciosa que cada una de aquellas. Aquí está el contenido, el misterio de la predestinación y la solución del problema Duo irregularia possunt aliquiado facere aliquid regulare [Dos cosas irregulares a veces pueden hacer algo regular]. Dios quiere la salud de todas las criaturas y lo que es mejor para ellas. (Teodicea, 1694-1698/2003b: 453)

Por otro lado, como ya de indicó, Leibniz considera que podemos imaginar un mundo posible sin pecado, sin enfermedad, pero este mundo es inferior al que vivimos ahora, por la misma razón ya expuesta, por que un mal o unos males pueden producir un bien mayor. Más adelante se verá que la existencia de la enfermedad lleva al conocimiento, en este caso de la enfermedad, la cual hace que los hombres orienten las ciencias a la salvaguarda de la vida, y con ello se logra el progreso de la humanidad en general. En sus palabras;

Es cierto que pueden imaginarse mundos posibles sin pecado ni desgracias y que se podría hacer con ellos novelas y utopías o Sevaramdas; pero esos mismos mundos serían muy inferiores en cuanto al bien respecto del nuestro. No puedo hacéroslo ver detalladamente, porque ¿acaso puedo conocer, y además representaros los infinitos, y compararlos entre sí? Vosotros debéis juzgarlo conmigo a partir de las consecuencias, por que Dios ha escogido este mundo tal y como es. Por otra parte, sabemos que a menudo un mal causa un bien, al que no se habría llegado sin este mal. Incluso muchas veces dos males han producido un bien [...] (Teodicea, 1710/2012: 101)

El enlace entre la salud y la enfermedad, en el marco del tema del mal en la Teodicea, de igual forma puede apreciarse en la siguiente cita, en la cual Leibniz asevera que la salud es considerada como un bien. Para él la salud debe ser lo ordinario, la enfermedad lo menos frecuente; empero, como ya se indicó,

Carvajal Villaplana, Álvaro (2021). «Leibniz; medicina, salud y epidemias». Monograma. Revista Iberoamericana de Cultura y Pensamiento, $\mathrm{n}^{\circ}$ 8, pp. 131-160. doi: 10.36008/monograma.2021.08.2003. http: revistamonograma.com. ISSN: 2603-5839. 
la atención reflexiva sobre la enfermedad conduce a una mejor valoración de la salud, la cual se enmarca en el contexto del principio de vida; la cita es reveladora:

Pero se dirá que los males son grandes y numerosos en comparación con los bienes; se engañan. No es sino la falta de atención lo que disminuye nuestros bienes, y es necesario que esta atención nos sea dada por alguna mezcla de males. Si estuviéramos enfermos habitualmente y rara vez sanos, sentiríamos extraordinariamente este gran bien, y sentiríamos menos nuestros males; pero ¿no vale más que la salud sea lo ordinario y la enfermedad lo raro? Suplamos, pues por nuestra reflexión lo que falta a nuestra percepción, para hacer más sensible el bien de la salud. Si no tuviéramos conocimiento de la vida futura, creo que encontraríamos a pocas personas que en el momento de la muerte no estuvieran contentas por recobrar la vida a condición de volver a pasar por los mismos bienes y males, siempre que sobre todo no fueran de la misma especie. Nos contentaríamos con variar, sin exigir una condición mejor que aquella en que habíamos vivido. (Teodicea, 1710/2012: 103)

La salud no solo está en conexión con la enfermedad, sino que también Leibniz la relaciona con la muerte. Al igual que las citas anteriores, si bien la enfermedad conforma parte de la armonía preestablecida y del mejor de los mundos posibles, Leibniz no se hunde en el pesimismo, sino que mantiene el optimismo, pues toda la acción humana va encamina a preservar la vida. En tal sentido se resiste a los enfoques pesimistas ante la muerte, por eso crítica a Montaigne, en el diálogo entre el sacerdote y el político ${ }^{7}$. En boca del sacerdote indica que Montaigne «[...] quiere que uno vaya a hundirse lejos de la muerte de cabeza

7 Diálogo entre un politico sagas y un sacerdote de reconocida piedad (1679, en Olaso, 2003).

Carvajal Villaplana, Álvaro (2021). «Leibniz; medicina, salud y epidemias». Monograma. Revista Iberoamericana de Cultura y Pensamiento, n. ${ }^{\circ}$ 8, pp. 131-160. doi: 10.36008/monograma.2021.08.2003. http: revistamonograma.com. ISSN: 2603-5839. 
baja y pensando en ella lo menos que pueda» (1679/2003, 258). Esto es como una especie de resignación a la muerte, como si estuviéramos «[...] en una prisión que nos ocasiona mil aflicciones, que suplicio mayor que la padecen los peores criminales [...]» (1679/2003: 258). Según él puede ser el caso que los condenados a muerte no sufran tanto como los que mueren por enfermedad (por la lenta agonía); aunque Leibniz no se cuestiona el sufrimiento que vive el condenado a muerte previo a su ejecución, ese tiempo de espera puede verse igualmente como una lenta agonía.

En todo caso, según él, hay algo más grave a lo que temer: los pensamientos tristes y oblicuos sobre el futuro. En sus palabras:

...en efecto, los decapitados no sienten casi nada al morir y la mayor parte de los que mueren en su lecho están sujetos a los tormentos de la agonía, que suelen superar a los que se infieren a los criminales. Pero todavía hay algo que temer más allá de la muerte, pues cualesquiera sean los esfuerzos que hagamos para distraernos (pour nous divertir) de la preocupación por el futuro, no está en nuestro poder impedir que se nos aparezcan pensamientos tristes y oblicuos que nos hacen pensar, pese a nosotros, en lo que vamos a ser, y que sirven de admonición a las personas corregibles, y de castigo a los malos, pues esa amargura salvífica para unos es insoportable para los demás [...] (1679/2003: 258)

Incluso, el político contraargumento al sacerdote que «[...] usted traza una terrible pintura de la vida y la muerte y para que me preocupe me cuenta cosas inverosímiles; pues todas esas bellas promesas se convierten en humano cuando uno las examina sin prevención y a menudo le he oído decir cosas a hipócritas o a visionarios. Lo cual me hace evitar en lo posible esas discusiones inútiles» (1679/2003: 259). El texto resalta la crítica a la perspec-

Carvajal Villaplana, Álvaro (2021). «Leibniz; medicina, salud y epidemias». Monograma. Revista Iberoamericana de Cultura y Pensamiento, $\mathrm{n}^{\circ}$ 8, pp. 131-160. doi: 10.36008/monograma.2021.08.2003. http: revistamonograma.com. ISSN: 2603-5839. 
tiva pesimista ante la muerte, así como a la perspectiva salvífica del sacerdote. En este escrito Leibniz critica el escepticismo, sin embargo, tal parece que «un poco» de escepticismo es permitido, la crítica parece que se dirige a un escepticismo radical.

La discusión entre el político y el sacerdote, aborda el método para llegar a resoluciones, una vez examinadas las cosas lo suficiente, de nuevo el escepticismo del político se posiciona ante el pensamiento salvífico del sacerdote, en especial cuando el último afirma que el escepticismo lleva a la incredulidad. A tal respecto, el político insiste en lo ignorante que somos de muchas cosas. Sin embargo, el sacerdote dice de los principios que son ciertos, por ejemplo, el principio de razón suficiente (el que es previo a la experiencia), del cual el político reconoce su certeza, pero el político insiste en que hay muchos asuntos sobre los que no tenemos certeza, por eso asevera que «[...] veo claramente que no es posible encontrar la razón de diversidad alguna cuando todo es semejante de un lado y de otro» (1679/2003: 264). El político reconoce que los principios son cosas muy seguras, pero que hay otras que son inseguras, como las incertidumbres del derecho, las contradicciones de los médicos, la diversidad de las costumbres (1679/2003: 265) ${ }^{8}$. Así, en los conocimientos prácticos — como la medicina— no contamos con certezas.

Los argumentos expuestos sobre la centralidad de la vida, la importancia de la salud, la no resignación ante la muerte y la enfermedad, así como la incertidumbre del conocimiento científico, en particular de la medica, conlleva al siguiente tema: el papel de las ciencias y de la medicina para el mantenimiento de la salud.

8 Ahora cuando el sacerdote y el político discuten sobre el razonamiento y el escepticismo, se presenta el debate sobre el error, sobre si este reside en la forma o en la materia. El político dice que el error está en la materia, aunque Leibniz insiste que es en la forma; en todo caso las proposiciones necesitan de la evidencia (1679/2003: 266-267).

Carvajal Villaplana, Álvaro (2021). «Leibniz; medicina, salud y epidemias». Monograma. Revista Iberoamericana de Cultura y Pensamiento, n. ${ }^{\circ} 8$, pp. 131-160. doi: 10.36008/monograma.2021.08.2003. http: revistamonograma.com. ISSN: 2603-5839. 


\section{Las ciencias para la acción: salud y medicina}

Leibniz piensa que existe una unidad de las ciencias, para alcanzar dicha unidad, él propone proyectos para el desarrollo de las ciencias. Las ciencias — al igual que la «verdadera religión»— tienen como fundamento la «jordenación universall», por lo que la razón puede servir para tal propósito; ya que, para él lo más grato es la armoniosa armonía de la naturaleza, por tales razones está convencido de que «[...] los espíritus eminentes, a quienes su posición se lo permita, deben obtener gran parte de su placer investigando las maravillas naturales y buscar las verdades magníficas y hermosas contenidas en las ciencias honestas [...]» (1669/2011: 445-446). Además, los conocimientos de las ciencias traen prestigio para el gobernante que los fomenta, en sus palabras: «[...] los bellos descubrimientos no solo traen gloria al gran señor que los fomenta, sino que acrecientan, además, el sustento de los súbitos, contribuyen a la comodidad de los hombres e incluso a la conservación de la salud. Pero independientemente de esto, arrojan tal luz sobre la obra principal de la naturaleza, y esto da origen a tal satisfacción» (1669/2011: 445446). Lo interesante de esta cita es que la investigación de las ciencias tiene como misión la conservación de la salud, así como sus resultados más fructíferos.

Las ideas de que las ciencias están conformes a la armonía universal, se constituyen en una unidad, y han de ser sometidas a prueba, lo expresa en el escrito Wilhelm Pacidius (Borrador de una introducción) (1671-1672), en el que Leibniz escribe bajo el sinónimo de Wilhelm Pacidius, ahí indica que

...y en el ejercicio de aquella libertad que él practicaba, se somete todo a prueba, había entrado en posición de una idea que, por cierto, no estaba elaborada. Sin embargo, como podía

Carvajal Villaplana, Álvaro (2021). «Leibniz; medicina, salud y epidemias». Monograma. Revista Iberoamericana de Cultura y Pensamiento, $\mathrm{n}^{\circ}$ 8, pp. 131-160. doi: 10.36008/monograma.2021.08.2003. http: revistamonograma.com. ISSN: 2603-5839. 
resultar adecuada para la armonía universal y la comprensión de los dominios de las ciencias que están relacionadas entre sí (dominios que se hallan coordinados en uno solo), se dedicó a reflexionar acerca de cuál era el mejor camino a seguir al respecto. (1671-1672/2011: 62)

De la cita cabe destacar que Leibniz establece la unión entre la teoría y la práctica de las ciencias, las cuales se orientan hacia el bien común, por ende, es algo que le corresponde a la medicina y la salud. La propuesta de que el conocimiento se orienta a la práctica, es decir, a la «mayor utilidad pública», y a lo que interesa al género humano, le corresponde realizarlos a los hombres, en particular al gobernante, pero no cualquier gobernante, sino uno ilustrado, lo cual se expresa de la siguiente manera:

[513] Por consiguiente, reflexionando sobre el pensamiento de mayor importancia para la vida, reflexionando como quien dice sobre una dirección (ratione) para el estado privado, establecía ante todo que, a una persona, en cuanto persona privada, debía parecerle finalmente lo mejor aquello que fuese de mayor utilidad pública, que correspondiere a la gloria de Dios y cuya ejecución interesara tanto a quien lo realiza como al género humano. Pero afirmaba que, para llevar a cabo cosas superiores para el hombre, ningún medio resultaba más notable que el propio hombre, y entre los hombres, el rey, vicario de Dios, ya sea por su poder como por su sabiduría, si la excepcional dicha de los tiempos nos depara un personaje con tales cualidades. (1671-1672/2011: 63)

De tal manera que en su escrito Consulta sobre la promoción del conocimiento del conocimiento de la naturaleza para su empleo en la vida y sobre la fundación de una sociedad con este fin (1676), Leibniz plantea un plan para la organización de la producción de conocimiento;

Carvajal Villaplana, Álvaro (2021). «Leibniz; medicina, salud y epidemias». Monograma. Revista Iberoamericana de Cultura y Pensamiento, n. ${ }^{\circ}$ 8, pp. 131-160. doi: 10.36008/monograma.2021.08.2003. http: revistamonograma.com. ISSN: 2603-5839. 
tal plan, y el conocimiento como tal, tiene que ordenarse a la práctica y a la solución de problemas (1676/2006: 370). Así:

La práctica, o sea, el fin de la investigación, se asienta en la praxis útil para la vida, o sea, en la solución de los problemas, cosas ambas de las que estamos necesitados. Necesitamos felicidad, o, lo que es lo mismo: tener el espíritu satisfecho; necesitamos de la virtud, de la salud, de los amigos, de los recursos de todo tipo; todas estas cosas dependen de nuestro conocimiento de Dios, del alma, del cuerpo, y de la aplicación de esta ciencia. (1676/2006: 370)

Como se señaló, Leibniz considera que en las ciencias no hay plena certeza, no se tienen todas las explicaciones causales de los efectos, por lo que en la práctica a veces puede existir una mala teoría, y de ahí la necesidad de promover la investigación científica. En Protogea, esta inquietud la expone Leibniz al hablar de las propiedades curativas las glossopetras, un antídoto contra los venenos, un medicamento en el ámbito de la medicina. Sin embargo, si bien tiene tales propiedades curativas, él ve que dichas propiedades pueden ser mal empleadas, en curaciones o en asuntos no apropiados. Leibniz está consciente de que las ciencias de su época, por ejemplo, la química no tienen explicaciones causales para los efectos, por lo que para él subsisten explicaciones míticas, por ende en la práctica subsiste la mala teoría. El tiene una intención pedagógica, ya que pretende desterrar la superchería y la credulidad (1691-1693/2006: 231-233).

La investigación en la ciencia, puede llevar al descubrimiento de enfermedades, se ha de destacar la referencia que hace a Helmont, un médico, físico y alquimista belga, al que se le considera padre de la bioquímica, y a Frederich Hoffman, un médico y alquimista alemán que descubrió enfermedades como la apen-

Carvajal Villaplana, Álvaro (2021). «Leibniz; medicina, salud y epidemias». Monograma. Revista Iberoamericana de Cultura y Pensamiento, $\mathrm{n}^{\circ}$ 8, pp. 131-160. doi: 10.36008/monograma.2021.08.2003. http: revistamonograma.com. ISSN: 2603-5839. 
dicitis y renovó el concepto de enfermedad (Teodicea, 1710/2012: $101)^{9}$. Estas son las ventajas de considerar que este es el mejor de los mundos posibles - y el que Dios escogiera-, ya que permite el progreso de la ciencia, y aumenta nuestro conocimiento.

Sobre concepciones erróneas de las enfermedades, Leibniz hace referencia a Boyle que rechaza a las cuatro cualidades como principios suficientes para explicar la naturaleza de las cosas, incluso como causa de la enfermedad. También cita a Hipócrates en su libro de medicina antigua, quien antes que Boyle las había rechazado, así expresa que «[...] las enfermedades llamadas de toda la sustancia, añadió Sennertus ser independientes de esas cuatro cualidades» (1687/2011: 56) de Empédocles, los peripatéticos y los galenistas.

Para Leibniz ${ }^{10}$ una vez establecida la autoridad de los sentidos, así como de otros testimonios, que fundamentan la historia de los fenómenos, puede formarse una ciencia mixta si se los combina, junto con las verdades extraídas de las experiencias. Pero, según él, es necesario «un arte especial»: «[...] tanto para hacer las experiencias como ordenarlas y combinarlas, a fin de que pueden hacerse a partir de ellas inducciones útiles y encontrar las causas y para que se establezcan los aforismos y los postulados (praenotiones) [...]» (1679/2011: 232). Sin embargo, reitera que la negligencia de los hombres descuida los asuntos importantes:

...pero es asombrosa la negligencia de los hombres, que gastan su tiempo en tonterías y descuidan aquello con que pueden procurarse la salud y el bienestar, ya que tendrían quizá a su disposición los remedios para gran parte de sus males si em-

9 Teodicea. Ensayos sobre la justicia de dios y la libertad del hombre en relación con el origen del mal (1710, en Vega, 2012).

10 Sobre la sintesis y el análisis universal, es decir, sobre el arte de descubrir y el arte de juggar (1679, en Echeverría, 2011).

Carvajal Villaplana, Álvaro (2021). «Leibniz; medicina, salud y epidemias». Monograma. Revista Iberoamericana de Cultura y Pensamiento, n. ${ }^{\circ}$ 8, pp. 131-160. doi: 10.36008/monograma.2021.08.2003. http: revistamonograma.com. ISSN: 2603-5839. 
plearan correctamente las numerosísimas observaciones que se han llevado a cabo en nuestro siglo el verdadero análisis. El conocimiento humano actual de la naturaleza que parece análogo a un comercio provisto copiosísimamente de toda clase de mercaderías, pero exento de orden e inventario. (1679/2011: 232)

En relación con el tema del progreso de las ciencias, y la creación de nuevas ciencias, la siguiente cita distingue en el proceso de la creación de una ciencia por medio del análisis, y la síntesis como el momento de su consolidación:

De lo dicho también resulta claro cuál es la diferencia entre la síntesis y el análisis. Se alcanza la síntesis cuando empezado desde los principios y recorriendo ordenamiento las verdades, aprendemos ciertas progresiones y establecemos algo así como tablas e incluso a veces fórmulas generales, en las que puede encontrar después lo que inicialmente se planteó [...] (1679/2011: 232)

A tal respecto, Leibniz señala como la salud es una de esas ciencias que requiere de las reflexión para eliminar el desconocimiento y la ignorancia:

De todo lo de este mundo, después de la tranquilidad del espíritu, no hay cosa más importante que la salud, cuya conservación, o recuperación, exige profundas meditaciones físicas y mecánicas. ¿Cuántas veces caemos en estado lastimoso por la sola ignorancia o inadvertencia de algún razonamiento fácil o de alguna observación obvia, que no se nos escaparía si nos aplicásemos de modo conveniente y si los hombres se sirviesen de sus ventajas [...] (1686/1999: 29).

La cita también resalta que la salud y la enfermedad no son solo un asunto de los aspectos físicos, sino también relativos a aspectos que podríamos considerar psicológicos o de «tranquilidad del espíritu» como él lo nombra. De la misma manera,

Carvajal Villaplana, Álvaro (2021). «Leibniz; medicina, salud y epidemias». Monograma. Revista Iberoamericana de Cultura y Pensamiento, n. ${ }^{\circ}$ 8, pp. 131-160. doi: 10.36008/monograma.2021.08.2003. http: revistamonograma.com. ISSN: 2603-5839. 
afirma que los estados del ánimo, influyen en las enfermedades: «[... se ha observado que los afectos del ánimo tienen mucha importancia en las enfermedades, cosa que no es de extrañar, pues los afectos agradables se acompañan siempre de movimientos convenientes, así como los violentos se acompañan de grandes movimientos [...]» $(1687 / 2011: 53)^{11}$.

En relación con las enfermedades psicológicas, Leibniz menciona, en el escrito La profesión de fe del filósofo, las enfermedades mentales, las que llama como «los insanos»; la que atribuye la «razón de los locos», a una razón arraigada en el temperamento, a la educación, etc. (1673/2003: 148).

Un último tipo de enfermedad, a las que refiere, son las «enfermedades espirituales» (Citado por Olaso, 2003, 240, 256): aquellas que están en relación - por ejemplo- con los que hacen «burlas al amor a la patria», o «ridiculizan a los que se preocupan por lo público» (2003: 240); empero estos pueden padecer los males que achacan a los demás, de tal manera que «[...] si nos corregimos ya de esta enfermedad espiritual epidémica cuyos malos efectos comienzan a ser visibles, acaso se impidan esos males; pero si sigue creciendo, la providencia corregirá a los hombres mediante la revolución misma que debe nacer de esa enfermedad» (2003: 240).

Las enfermedades mentales, las psicológicas y las del espíritu no se estudian con detalle en este artículo, sin embargo, es valioso destacar la recomendación general que hace Leibniz para evitar tales enfermedades. Según la siguiente cita el hastío y la desesperanza, —en general los estados de ánimo— son formas de dañar la salud del alma:

11 El antibarbarus phisicus en defensa de la filosofía real contra la renovación de las cualidades y las inteligencias quiméricas (1687, en Andreu, 2011).

Carvajal Villaplana, Álvaro (2021). «Leibniz; medicina, salud y epidemias». Monograma. Revista Iberoamericana de Cultura y Pensamiento, n. ${ }^{\circ}$ 8, pp. 131-160. doi: 10.36008/monograma.2021.08.2003. http: revistamonograma.com. ISSN: 2603-5839. 
...hay que buscar todos los recursos imaginables para moderar las pasiones que pueden perturbar el uso de la razón. Por esto hay que acostumbrarse a no irritarse por nada, a no encolerizarse en absoluto, a evitar toda tristeza, lo que se puede hacer cuando uno está bien persuadido de nuestras grandes verdades. En lo que respecta a la alegría, solo debe ser moderada y pareja; pues una gran expansión de los espíritus es seguida por una natural tristeza y daña mucho la salud. Después de una alegría moderada no hay pasión más hermosa y más útil que la esperanza; o mejor, esa alegría pareja y perdurable solo consiste en una esperanza bien fundada [...] (1679/2003: 284-285)

Una vez examinada la relación de la medicina con la enfermedad, ahora corresponde verla desde otra óptica, la justicia y el bien común.

\section{Salud: principio de equidady bien común}

En el escrito La suprema regla del derecho (1678), Leibniz se refiere al bien común, como el valor fundamental de su concepción política. Asimismo, establece una serie de bienes útiles, los cuales tienen que estar a disposición y distribuidos entre muchos, «[... en vez de pertenecer en grado sumo a unos pocos [...]» (1678/2001: 535). Entre algunos de esos bienes están: «[...] todos los ciudadanos han tener ánimo tranquilo y satisfecho, sean moderados, prudentes, estén bien dispuestos hacia el bien común, han de ser piadosos, que amen y honren a los gobernantes, que sean amigos entre sí, que sepan muchas cosas [...]» (1678/2001: 536). Leibniz parece pedir demasiado, con esta idea de «amar a los gobernantes», es claro que existen gobernantes no ilustrados, corruptos y totalitarios que no merecen dicho amor.

Leibniz considera que la condición física es producto o está influida por la condición mental, lo mismo indica sobre la in-

Carvajal Villaplana, Álvaro (2021). «Leibniz; medicina, salud y epidemias». Monograma. Revista Iberoamericana de Cultura y Pensamiento, $\mathrm{n}^{\circ}$ 8, pp. 131-160. doi: 10.36008/monograma.2021.08.2003. http: revistamonograma.com. ISSN: 2603-5839. 
fluencia de la belleza que genera una disposición positiva hacia las personas, en sus palabras: «[...] sean de cuerpo elegante, tengan salud y sean ágiles; la razón de ello es que el cuerpo debe ejecutar lo que la mente decida. Por otra parte, la belleza influye mucho en la disposición de ánimo que los demás tienen hacia nosotros» (1678/2001: 537). De tal manera, que para Leibniz las «virtudes físicas y morales» han de ser consideradas como bienes que se han de ejercitar. Por otra parte, es preciso que los seres humanos «[...] tengan cubiertas las necesidades básicas de la vida, porque es sabido que la pobreza hace a los hombres desgraciados y malvados» (1678/2001: 537). En definitiva, tiene que haber los suficientes medios para que todos puedan hacer el bien.

Para Leibniz los bienes son útiles o necesarios, llama bienes necesarios "[...] a aquellos que se requieren para la tranquilidad del ánimo, o aquellos cuya ausencia nos aflige [...]» (1678/2001, 137). Los útiles son «[...] todo lo demás, las cosas de las que prescindimos con facilidad [...]» (1678/2001: 137). Pero los bienes necesarios tienen mayor importancia que los útiles (1678/2001: 137).

Según como lo expone en el escrito Suprema regla del derecho (1678), el bien común es «[...] la suma de los bienes de cada individuo; por consiguiente, diremos que el mayor bien común consiste en que sea lo mayor posible y lo más grande posible el número de bienes que cada uno obtiene o que a cada uno caben en suerte» (1678/2001: 137). Aquí vincula el bien común, con la adquisición de esos bienes o los que atribuye la suerte. Sin embargo, si consideramos el principio de equidad, así como otros textos citados en este artículo, la salud como bien común sería el más distribuido. Para Leibniz el principio indica que «[...] es el principio de equidad o, lo que es lo mismo, de igualdad, el que exige que se conceda a otro lo que uno pretendería en el caso

Carvajal Villaplana, Álvaro (2021). «Leibniz; medicina, salud y epidemias». Monograma. Revista Iberoamericana de Cultura y Pensamiento, n. ${ }^{\circ} 8$, pp. 131-160. doi: 10.36008/monograma.2021.08.2003. http: revistamonograma.com. ISSN: 2603-5839. 
de estar en parecidas circunstancias, sin aspirar a un privilegio en contra de la razón, o poder alegar la voluntad propia como razón» (1703/2006: 93).

En especial, según él, tal principio demanda que se haga el bien cuando sea oportuno, y a cada uno lo que le corresponde, lo cual expone en Meditación sobre la noción común de justicia. A este respecto, la salud entendida como un bien común, es más importante que cualquier costo, en sus palabras: «[...] en lo relativo a la salud, no ha de tenerse en cuenta consideración monetaria» (1669-1672/1991: 28); salvo en las consideraciones que se plantean por razones monetarias en el caso de Cayo ${ }^{12}$, en donde media un costo y un cálculo, ya que en una guerra puedo elegir a quién socorrer en primer lugar sobre la base de un cálculo.

Estas reflexiones sobre la salud como un bien común, en donde prima la justicia, y el reparto de las necesidades básicas, permiten el paso al punto de visto ético que rige las recomendaciones y las medidas sugeridas por Leibniz para prevenir y contener una epidemia.

\section{Pandemias, prevención y medidas sanitarias}

Considerando la importancia que Leibniz le otorga a la salud, en tanto un bien común, algo que está al mismo nivel de importancia que la razón; además, que se trata, a la vez, de un medio cuya finalidad es llevarnos a la felicidad; no es de extrañar que preste especial atención a la crisis que produce una pandemia. En contraste con las reflexiones de Leibniz sobre la salud, las cuales hubo que rastrearlas por buena parte de su obra, teniendo que hacer una reconstrucción de sus ideas; en el caso de las pan-

12 Véase Elementos de derecho natural (1669-1673/1991).

Carvajal Villaplana, Álvaro (2021). «Leibniz; medicina, salud y epidemias». Monograma. Revista Iberoamericana de Cultura y Pensamiento, n. ${ }^{\circ}$ 8, pp. 131-160. doi: 10.36008/monograma.2021.08.2003. http: revistamonograma.com. ISSN: 2603-5839. 
demias, el autor sistematiza su teoría sobre el abordaje de una pandemia en la carta al Duque Ernst-Augusto (1681).

Para Leibniz acometer el tema de las pandemias no es un solo un asunto de interés teórico, sino más bien ético y político, ya que $«[. .$.$] considera que la prudencia y el deber exigen que$ considere con detenimiento lo que debe hacerse, para no reprocharnos, ante una epidemia» (1681/2006: 227). Él considera que las medidas preventivas y de contención que presenta en dicha carta son el resultado de la observación de otros casos de pandemias (1681/2006: 229); luego por la urgencia de atender una, la cual pone en peligro a su país (el caso de Nordhausen), de ahí la recomendación que hace al Duque a Ernst-Augusto.

Para Leibniz, la contención de las pandemias tiene que ver con la acción humana, no obstante, recurre a Dios como fuente de inspiración para dicha acción, de tal manera que Dios «[...] compadecido por nuestras súplicas, nos dará las luces necesarias para impedir el progreso del mal [...]» (1681/2006: 227). Sin embargo, si bien la acción humana es la responsable de prevenir la pandemia, y aunque se tomen todas las medidas necesarias, puede ser que el «[...] buen Dios, tenga a bien castigarnos, y la epidemia llega al país, hay que seguir trabajando para nuestra salvación [...]» (1681/2006: 231).

Este énfasis en las acciones humanas, más que en la intervención divina, me parece es lo que lleva a firmar a Leibniz que en las pandemias «[...] muchas personas que podrían salvarse por la peste, perecen por falta de una buena organización, de modo que el problema se reduce a estas dos cuestiones: la prevención y la curación» (1681/2006: 227). En este sentido, la estrategia de intervención en la pandemia conforma parte de una perspectiva ética, la que está centrada en la acción política que asume el gobernante, de ahí el por qué dirige la carta al Conde.

Carvajal Villaplana, Álvaro (2021). «Leibniz; medicina, salud y epidemias». Monograma. Revista Iberoamericana de Cultura y Pensamiento, n. ${ }^{\circ} 8$, pp. 131-160. doi: 10.36008/monograma.2021.08.2003. http: revistamonograma.com. ISSN: 2603-5839. 
Si bien, Leibniz considera que pueden tomarse todas las medidas necesarias, es posible que una estrategia contra la pandemia tenga inconvenientes o errores (aunque puede aprenderse de los errores, dice Leibniz), en razón de que «[...] la debilidad humana, la negligencia, la imprudencia, la malicia de algunos para escapar de un lugar peligroso, la falsa caridad de otros, el estado de opinión injusto, pero peligroso, que desaprueba las precauciones tomadas contra la peste. Todo esto hace que las acciones no se lleven a cabo con demasiada precisión» (1681/2006: 229).

Como ya se indicó, Leibniz cree que la medicina no puede basarse en la superstición, sino que ha identificarse las causas de los efectos, en el caso de las pandemias, como los médicos todavía «[...] no han encontrado medidas preventivas seguras, hay que recurrir a medidas de tipo político para evitar el contagio, que es lo único eficaz [...]» (1681/2006: 227). Se interpreta que los médicos no tienen una cura o un tratamiento efectivo para combatir la enfermedad. A pesar de ese desconocimiento, él sabe que un contagio se «[...] produce por el aire, el contacto entre hombres y animales, o las cosas que se hayan tocado, por el contacto con las cosas en el aire infectado a podido penetrar [...]» (1681/2006: 227). Él habla de sustancias peligrosas que quedan impregnadas en los tejidos o las materias grasas. Tales sustancias peligrosas no vienen por sí mismas, «[...] sino que habitualmente las traen personas muy sospechosas [...]» (1681/2006. 228). Por tal razón, se han de dirigir la acciones de vigilancia hacia los hombres.

En su estrategia de control y prevención, Leibniz sigue dos etapas: a) la primera cerrar las fronteras del país, y b) si el contagio ha pasado la frontera se sigue una estrategia al interior. En la primera etapa, la vigilancia y el mantenimiento del aislamiento entre la personas puede ser dos tipos: «[...] general o particular.

Carvajal Villaplana, Álvaro (2021). «Leibniz; medicina, salud y epidemias». Monograma. Revista Iberoamericana de Cultura y Pensamiento, $\mathrm{n}^{\circ}$ 8, pp. 131-160. doi: 10.36008/monograma.2021.08.2003. http: revistamonograma.com. ISSN: 2603-5839. 
La general se ejerce sobre toda la comunidad y la región; la particular, atendiendo a algunas personas y casas [...]» (1681/2006: 228). En la general, se trata de una vigilancia de ingresos y salidas a la región, la cual puede ser fija y móvil (1681/2006: 228) ${ }^{13}$.

Otra media en esta etapa consiste en intervenir en una región en la que hay peste, en donde los vecinos no tomaron las medidas preventivas, esto para proteger a esas personas, a su pesar. Para ello dice Leibniz habría que mandar tropas, como en el caso de Nordhausen, un pueblo «[...] que está en peligro y que ahora nos afecta [...]» (1681/2006: 228-229).

Empero, si a pesar de tales medidas, algunos infectados logran atravesar los controles, y con ello extiende la peste a la región que se pretende proteger, es decir si «[...] se ha extendido [la peste] a los cuerpos de quienes eran muy propensos al mal [...]» (1681/2006: 229); razón por lo cual, Leibniz considera que el control deber ser riguroso, e incluso, se ha de asegurar que a los que escapan del control no encuentren refugio en otro lugar (1681/2006: 229). Además, los infractores deben ser castigados (1681/2006: 230). En esta etapa, asimismo parece que Leibniz se referirse a la cuarentena de aquellas «[...] personas del lugar que hayan estado ausentes un espacio de tiempo suficiente como para ir a un lugar sospechoso, tendrá que ser examinados al volver [...]» (1681/2006: 230).

Una vez que la peste ha llegado a la región, las medias de control o autocontrol deben ser ejercidas por el padre de familia

13 Detalles sobre la vigencia de las fronteras: «[...] la guardia fija es la que hay en los puestos, es decir, que debe apostarse en los caminos y en las puertas. Además, centinelas situados en lugares altos, para describir desde lejos a los que pudieran venir del lado donde no se quiere que venga nadie [...]» (1681/2006: 228). «La guardia móvil es también doble: en campos raso habrá algunas unidades de caballería, que batirá el terreno; en los bosques y montañas será necesario que monten guardia los cazadores, y reconozcan los lugares [...]». Todo esto servirá para cerrar los caminos (1681/2006: 228).

Carvajal Villaplana, Álvaro (2021). «Leibniz; medicina, salud y epidemias». Monograma. Revista Iberoamericana de Cultura y Pensamiento, n. ${ }^{\circ} 8$, pp. 131-160. doi: 10.36008/monograma.2021.08.2003. http: revistamonograma.com. ISSN: 2603-5839. 
o los ciudadanos en sus casas, por lo que no deben recibir personas sospechosas en sus casas (1681/2006: 230).

Todas las medidas han de ser publicadas en un decreto (1681/2006, 230), así como las sanciones y los castigos físicos.

Para Leibniz no es suficiente con establecer la ley de la situación de emergencia, así como las medidas a tomar; a la vez que es necesario su divulgación, y la suministración de información de las medidas, esto, se entiende para evitar «[...] la creencia en una predestinación absurda o la falsa caridad» (1681/2006: 230).

Leibniz está consciente de que en épocas de crisis como una peste, se pueden dar abusos de poder, por eso considera que deben tomarse medidas de protección del abuso de poder por parte de las autoridades, en este caso los magistrados menores (1681/2006: 231).

Por otra parte, si a pesar de que la primera barrera de protección no sea efectiva, y el contagio se extiende al interior del país, se ha de

...proteger la parte del país que aún esté incólume, del mismo que antes se guardó al país entero; por la misma razón se intentará aumentar más la vigilancia, ya que la experiencia nos habrá mostrado para entonces los errores que se hubieran cometido. Se procurará que, en caso de necesidad, no falten la madera, ni los medicamentos, ni los médicos, ni otras cosas necesarias. Cuando la peste ya haya invadido alguna calle en una gran ciudad entonces se la bloqueará o cerrará separándola del resto de la ciudad con cadenas y otros medios [...] (1681/2006: 231)

Además, se tomarán otras medidas, por ejemplo, las casas infectadas quedarán clausuradas, y sus habitantes permanece-

Carvajal Villaplana, Álvaro (2021). «Leibniz; medicina, salud y epidemias». Monograma. Revista Iberoamericana de Cultura y Pensamiento, $\mathrm{n}^{\circ}$ 8, pp. 131-160. doi: 10.36008/monograma.2021.08.2003. http: revistamonograma.com. ISSN: 2603-5839. 
rán en ella. Leibniz afirma que «[...] se harán visitas para ver que necesitan, para que les falte lo menos posible, y llevárselo» (1681/2006: 231). Las personas que hacen las visitan no deben recibir por parte de los afectados nada a cambio, esto suponemos para evitar la corrupción.

En momentos de cuarentena, y a partir de la aplicación del principio de equidad, Leibniz considera que aquellas personas que puedan tener empleados que atiendan sus necesidades que los sigan contratando. En contraste, a los pobres se les tendrá que pagar a alguien para que atiendan sus necesidades, para que las personas de la casa tengan sus previsiones. Otra disposición de ayuda económica, consiste en que se darán créditos «[...] allí donde exista alguna posibilidad de cobrarlos [...]» (1681/2006: 232). Pero cuando no exista tal posibilidad, «[...] toda la comunidad ayudará a soportar la carga de algunos de sus miembros, ya que es preciso que lo que a algunos sobra cubra la necesidad de otros. Los que tengan con qué, podrán abstenerse con prontitud de la mayor parte de las cosas necesarias [...]» (1681/2006: 232).

Asimismo, dice Leibniz que cuando las «[...] personas que viven al día de su jornal, serán ayudados, procurándose la venta de sus productos, sin necesidad de que ellos tengan que salir frecuentemente» (1681/2006: 232).

En el aspecto productivo afirma que aquellos que elaboran productos de necesidad, seguirán son sus trabajos, tomando las precauciones del caso. Él dice que se puede crear un sistema de distribución de los productos.

Para Leibniz los lugares más peligrosos para las pandemias son las ciudades, por lo cual él recomendó que la gente viaje al campo, esta es una medida que sea ha visto que con la pandemia de la COVID-19 no es efectiva, sino contraproducen- 
te. Así, los edificios que queden desocupados pueden usarse como hospitales (1681/2006: 232). En las ciudades pueden establecerse lugares de aislamiento para personas en cuyas casas esté bajo la infección, y que se pueda comprobar que no están contaminados, de esos lugares. Sin embargo, de tales sitios (hospitales provisionales) no podrán salir libremente; empero él considera que ahí estarían mejor que en sus casas infectadas (1681/2006: 233).

Por último, Leibniz hace una serie de recomendaciones, que, si bien no todas podrían considerarse científicas, si tienen una función o sirven para mantener el buen ánimo durante la cuarentena; algunos de tales recomendaciones coadyuvan a evitar la depresión; tal como hoy se haría sugerencias en una línea de pensamiento como la de Leibniz.

El aire, los perfumes de enebro, el agua fresca, la ropa blanca, los lechos suficientemente ventilados, los baños y las saunas, el jugo de limón, la música y un poco de vino para animar y recrear los espíritus, todo ello podrá mantener sanos a algunos, y salvar a los enfermos. No dudo de que todas estas medidas, y otras parecidas, podrán frenar mucho el avance de esta epidemia, contra la cual no se ha encontrado un eficaz antídoto (1681/2006: 233).

Con esta cita final, podemos ver cómo Leibniz no solo pensó en la protección de la salud de las personas, también mostró su preocupación por la situación económica de la comunidad durante la pandemia, así como la salud mental o el bienestar general de la población que se encuentra en una cuarentena por el distanciamiento que se ha de tener. La actitud de ética de Leibniz es sorprende, y frente al dilema que presenta una pandemia el ofrece su propuesta. Su pensamiento se muestra muy actual.

Carvajal Villaplana, Álvaro (2021). «Leibniz; medicina, salud y epidemias». Monograma. Revista Iberoamericana de Cultura y Pensamiento, $\mathrm{n}^{\circ}$ 8, pp. 131-160. doi: 10.36008/monograma.2021.08.2003. http: revistamonograma.com. ISSN: 2603-5839. 


\section{REFERENCIAS BIBLIOGRÁFICAS}

OBRAS DE LEIBNIZ

LeIBnIZ, G. W. (1710/2012). Teodicea. Ensayos sobre la justicia de dios y la libertad del hombre en relación con el origen del mal. En GuILLÉN VERA, Tomás (ed.). G. W. Leibniz. Obras filosóficas y cientificas. Granada, España: Comares.

- (1704/2011). «Consideraciones sobre los principios de vida y sobre las naturalezas plásticas, por el autor del sistema de la armonía preestablecida» (1705). En Leibniz, estudios y selección de Javier Echeverría. Madrid: Gredos.

(1703/2006). «Meditación sobre la noción común de justicia». En Escritos de filosofía jurídica y política. Edición de Jaime de Salas. Madrid: Biblioteca Nueva, pp. 81-103.

- (1691-1693/2006). Protogaea. Del primitivo aspecto de la tierra y su antiquísima historia según los vestigios de los propios monumentos de la naturaleza. Oviedo, España: KRK.

— (1687/2011). «El antibarbarus phisicus en defensa de la filosofía real contra la renovación de las cualidades escolásticas y las inteligencias quiméricas». En Andreu, Agustín. Methodus Vitae (Escritos de Leibniz). Naturaleza o fuerza, vol. I. Valencia: Universidad Politécnica de Valencia, pp. 53-60.

— (1686/1999). «La organización del conocimiento y la felicidad del género humano». En Andreu, Agustín. Methodus Vitae (Escritos de Leibniz). Naturaleza o fuerza, vol. I. Valencia: Universidad Politécnica de Valencia, pp. 3-16. - (1681/2006). «Carta de Leibniz al Duque Ernst-Augusto». En Escritos de filosofía jurídica y política. Edición de Jaime de Salas. Madrid: Biblioteca Nueva, pp. 227-233.

— (1679/2011). «Sobre la síntesis y el análisis universal, es decir, sobre el arte de descubrir y el arte de juzgar». En OLAso, 
Ezequiel de (ed.). Gottfried Wilhelm Leibniz: Escritos Filosóficos. Madrid: Machado Libros, pp. 39-47.

_ (1679/2003). «Diálogo entre un político sagaz y un sacerdote de reconocida piedad». En Olaso, Ezequiel de (ed.). Gottried Wilhelm Leibniz. Escritos Filosóficos. Madrid: Machado Libros, pp. 253-291.

(1678/2001). «La suprema regla del derecho». En Escritos de filosofía jurídica y política. Edición de Jaime de Salas. Madrid: Biblioteca Nueva, pp. 137-139.

(1677/2003). «Diálogo entre un teólogo y un misósofo».

En Olaso, Ezequiel de (ed.). Gottfried Wilhelm Leibniz: Escritos Filosóficos. Madrid: Machado Libros, pp. 243-252.

— (1676/2006). «Consulta sobre la promoción del conocimiento de la naturaleza para su empleo en la vida y sobre la fundación de una sociedad con este fin». En Escritos de filosofía jurídica y política. Edición de Jaime de Salas. Madrid: Biblioteca Nueva, pp. 351-373.

—_ (1673/2003). «La profesión de fe del filósofo». En Olaso, Ezequiel de (ed.). Gottfried Wilhelm Leibniz: Escritos Filosóficos. Madrid: Machado Libros, pp. 115-169.

— (1671-1672/2011). «Wilhelm Pacidius (Borrador de una introducción». En Olaso, Ezequiel de (ed.). Gottfried Wilhelm Leibniæ. Escritos Filosóficos. Madrid: Machado Libros, pp. 59-63. (1669-1672/1991). Elementos de derecho natural. Madrid: Tecnos. - (1669/2011). «Para una balanza del derecho que permita apreciar los grados de las pruebas y de las probabilidades». En Olaso, Ezequiel de (ed.). Gottfried Wilhelm Leibnir. Escritos Filosóficos. Madrid: Machado Libros, pp. 428-433.

(1694-1698/2003c). «De la felicidad». En Olaso, Ezequiel de (ed.). Gottfried Wilhelm Leibniæ. Escritos Filosóficos. Madrid: Machado Libros, pp. 455-465.

Carvajal Villaplana, Álvaro (2021). «Leibniz; medicina, salud y epidemias». Monograma. Revista Iberoamericana de Cultura y Pensamiento, n. ${ }^{\circ}$ 8, pp. 131-160. doi: 10.36008/monograma.2021.08.2003. http: revistamonograma.com. ISSN: 2603-5839. 
(1694-1698/2003b). «De la verdadera teología mística. En

Olaso, Ezequiel de (ed.). Gottfried Wilhelm Leibniæ. Escritos Filosóficos. Madrid: Machado Libros, pp. 449-454.

(1694-1698/2003a). «De la Sabiduría». En Olaso, Ezequiel

de (ed.). Gottfried Wilhelm Leibniz: Escritos Filosóficos. Madrid:

Machado Libros, pp. 461-465.

_ (1989). Filosofia para princesas. Madrid: Alianza.

\section{OBRAS SOBRE LEIBNIZ}

Andreu, Agustín. Methodus Vitae (Escritos de Leibniø). Naturaleza o fuerza, vol. I. Valencia: Universidad Politécnica de Valencia. Arana, Juan (ed.) (2009). G. W. Leibniæ: Obras filosóficas y cientifica. Escritos cientificos. Tomo 8. Granada, España: Comares.

Cardona SuÁres, Luis Fernando (2012). Dolor en la armonía. Justificación leibniz̨iana del sufrimiento. Granada, España: Comares.

Carvajal Villaplana, Álvaro (2016). «Muerte, sufrimiento y mal en Leibniz». En Nicolás, J. A. et al. La monadología de Leibniza a debate. Granada, España: Comares, pp. 225-238.

(2019). «Leibniz: argumentos para la defensa de los animales». En Carvajal, Álvaro (ed.). Leibni\%. Máquinas inteligentes, multiculturalismo y ética de la vida. Granada, España: Comares, pp. 45-69.

ECHEVERRíA, Javier (1992). «Introducción». En LeIBNIZ, G. W. Nuevos ensayos sobre el entendimiento bumano. Madrid: Alianza.

- (2009). Leibniæy su obra. México: UNAM. - (2011). «Estudio Introductorio». En EcheVERría, J. (ed.). Leibniæ: Madrid: Gredos.

Nicolás, Juan Antonio (2013). «Leibniz: de la biología a la metafísica vitalista». En AranA, Juan (ed.). Leibnizy las ciencias. Madrid: Plaza y Valdés/Sociedad Española Leibniz, pp. 179-209.

Carvajal Villaplana, Álvaro (2021). «Leibniz; medicina, salud y epidemias». Monograma. Revista Iberoamericana de Cultura y Pensamiento, n. ${ }^{\circ}$ 8, pp. 131-160. doi: 10.36008/monograma.2021.08.2003. http: revistamonograma.com. ISSN: 2603-5839. 
Olaso, Ezequiel de (ed.) (2003). Leibniz, G. W.. Gottfried Wilhelm Leibniz: Escritos Filosóficos. Madrid: Machado Libros.

RoBIneT, André (2005). Justicia y terror. Leibnizy el principio de la razón. Valencia: Universidad Politécnica de Valencia.

Roldán, Concha (2013). "Teodicea y libertad en Leibniz: del mal radical al bien común». En: SAn Martín, J.; SÁNchez, J. J. (eds.). Pensando la religión, Madrid: Trotta/UNED, pp. $448-461$.

Rovira, Rogelio (2006). Léxico fundamental de la metafísica de Leibniz. Madrid: Trotta.

Carvajal Villaplana, Álvaro (2021). «Leibniz; medicina, salud y epidemias». Monograma. Revista Iberoamericana de Cultura y Pensamiento, $\mathrm{n}^{\circ}$ 8, pp. 131-160. doi: 10.36008/monograma.2021.08.2003. http: revistamonograma.com. ISSN: 2603-5839. 